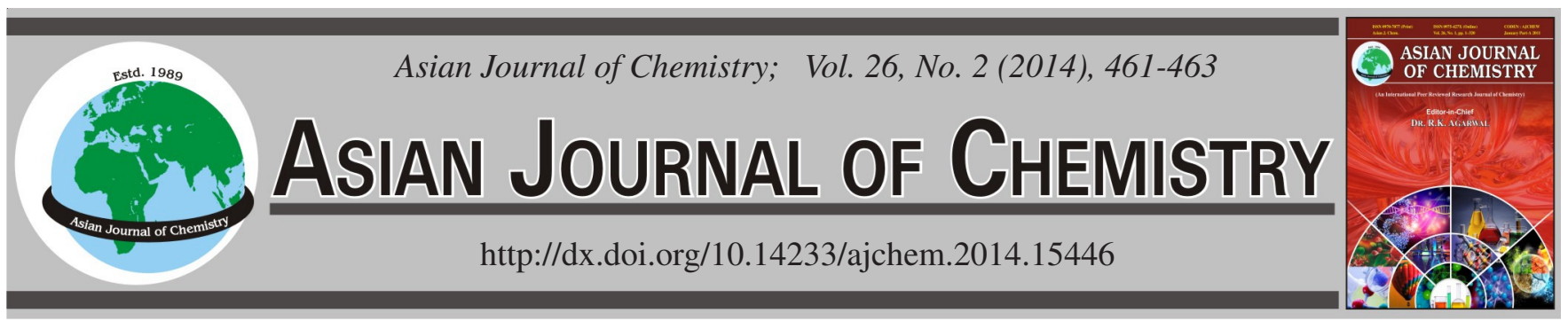

\title{
Variation of Carotenoid Content in Different Bitter Melon Cultivars
}

\author{
SAng Won Lee ${ }^{1}$ J Je Kwang Kim², Md. Romij Uddin ${ }^{3}$, Yeon BoK Kim³ \\ Haeng Hoon Kim ${ }^{4}$, Eunsook Chung ${ }^{5}$, JaI-HeOn LeE ${ }^{5, *}$ and SANG Un PARK ${ }^{3, *}$
}

\begin{abstract}
${ }^{1}$ Herbal Crop Research Team, National Institute of Horticultural \& Herbal Science, RDA, Eumseong-gun, Chungcheongbuk-do 369-873, Republic of Korea

${ }^{2}$ Division of Life Sciences, College of Life Sciences and Bioengineering, Incheon National University, Incheon, 406-772, Republic of Korea ${ }^{3}$ Department of Crop Science, Chungnam National University, 99 Daehangno, Yuseong-gu, Daejeon, 305-764, Republic of Korea

${ }^{4}$ Department of Well-being Resources, Sunchon National University, 413 Jungangno, Suncheon, Jeollanam-do, 540-742, Republic of Korea

${ }^{5}$ Department of Genetic Engineering, Dong-A University, Busan 604-714, Republic of Korea
\end{abstract}

*Corresponding authors: Fax:+82 51 2007505; Tel: +82 51 2007592; E-mail: jhnlee@dau.ac.kr; supark@cnu.ac.kr

\begin{abstract}
Nine different bitter melon cultivars collected from Japan and Philippines were analyzed to distinguish the levels of three different carotenoids i.e., lutein, $\alpha$-carotene and $\beta$-carotene using HPLC. The carotenoid content varied significantly among the cultivars from the 2 locations. In general, the carotenoid contents in the Philippines cultivar was higher than in the Japanese ones, with the highest content found in Galaxy from Philippines and the lowest in the cultivar Kyushu from the Japanese cultivar. The levels of lutein in the Galaxy cultivar were 2.9 and 2.8 times higher than Kyushu and Trident 357 cultivars, respectively. The cultivar Galaxy from Philippines contained 3.7, 3.1 and 3.0-fold higher $\alpha$-carotene compared to Kyushu, Sta. Rita Strain. L and Peacock, respectively. The levels of $\beta$-carotene in the Galaxy cultivars were 3.9, 2.9 and 2.8 times higher than that of Kyushu, Sta. Rita Strain. L and Peacock, respectively. The results suggest that the bitter melon cultivar Galaxy could potentially be used as a source of carotenoid.
\end{abstract}

Keywords: Bitter melon, Carotenoid, Cultivars, Geographical location.

\section{INTRODUCTION}

Bitter melon (Momordica charantia L.) is a medicinal plant indigenous to temperate and tropical regions of Asia and to other areas in the world belongs to the family Cucurbitaceae. The fruit of $M$. charantia is commonly known as the bitter melon or the bitter gourd because of its bitter taste. The fruit is oblong and resembles a cucumber. The fruit is emerald green when young and turns to orange-yellow upon ripening ${ }^{1,2}$. Now a days in many countries, bitter melon is used for the treatment of diabetes and colic and as a carminative ${ }^{3-5}$. The fruit and seeds of bitter melon are traditionally used as medicinal herbs for their antiHIV, antiulcer, antiinflammatory, antileukemic, antimicrobial, antidiabetic and antitumor properties ${ }^{6-10}$.

Carotenoids are a diverse group of more than 600 naturally occurring red, orange and yellow pigments ${ }^{11}$ that accumulate in the plastids of leaves, flowers and fruits. These pigments play important roles in many physiological processes in plants. For example, some act as light absorbers in photosynthetic membranes and prevent damaging photo-oxidative processes ${ }^{12}$ ). In addition, the colours of carotenoids in flowers and fruits attract pollinators and seed-dispersal agents ${ }^{13}$. In humans, some carotenoids are essential nutrients, while others have protective effects against several diseases. For example, provitamin A carotenoids such as $\alpha$-carotene and $\beta$-carotene, which are precursors of vitamin A, are necessary to prevent xerophthalmia, blindness and premature death ${ }^{14}$. The carotenoid composition may varies with variety, culture, cultivation conditions, the state of maturity, the post-harvest and storage handling, the climate and the geographical localization, the type of sample and the part of plant ${ }^{15,16}$.

Keeping in view the importance of carotenoids and their variation due to different parameters especially due to variety/ cultivars and geographical localization, reliable assessments of carotenoids in bitter melon used as vegetable is necessary for accurate quantification of dietary intake. Since no study on carotenoids determination in bitter melon by high performance liquid chromatography has been done yet, the present study was designed to determine cultivars from different sources in order to assess the carotenoid levels of the bitter melons cultivars. 


\section{EXPERIMENTAL}

Seeds of 9 bitter melon cultivars (4 from Japan and 5 from the Philippines) were germinated and then the seedlings were transferred to the greenhouse maintain the temperature at $25^{\circ} \mathrm{C}, 60 \% \mathrm{RH}$ and allowed to normal day light condition in experimental farm at Chungnam National University (Daejeon, Korea). After 4 months (at green edible stage), the fruits of 9 bitter melon cultivars were harvested and used to compare their carotenoid contents.

Carotenoid extraction: Samples (approximately $1 \mathrm{~g}$ ) were extracted at room temperature with $20 \mathrm{~mL}$ of hexane to remove the lipids and then dried in the hood. The procedure was repeated twice. Samples were then extracted with $10 \mathrm{~mL}$ of $\mathrm{MeOH}(100 \%)$ in a Sonicator (Branson Ultrasonic Co., Danbury, CT, USA) for $1 \mathrm{~h}$. The extract was filtrated through filter paper (Whatman No. 42) and evaporated (Heidoph VV2011, $\left.40{ }^{\circ} \mathrm{C}\right)$. The evaporated extract was re-suspended with $1 \mathrm{~mL}$ of $\mathrm{MeOH}$ for HPLC analysis.

HPLC analysis of carotenoid: The charantin was analyzed using an HPLC system (NS-4000; Futecs Co., Daejeon, Korea) with a UV detector (204 nm). The separation was performed on an Optimapak $(4.6 \mathrm{~mm} \times 250 \mathrm{~mm}, 5 \mu \mathrm{m}$, 100; RStech, Korea) with a flow rate of $0.8 \mathrm{~mL} \mathrm{~min}^{-1}$. The mobile phase used was $98 \% \mathrm{MeOH}$ and $20 \mu \mathrm{L}$ was injected for each sample. Identification and quantification of charantin was carried out by comparing the retention times and the peak areas, respectively, with standards or by the direct addition of the standard into the sample (spike test). Sample aliquots were filtered through a $0.45 \mu \mathrm{m}$ poly (tetrafluoroethylene) filter prior to injection. All samples were run in triplicate. Standard chemicals were purchased from Chromadex Inc. (Santa Ana, Calif., USA).

Statistical analysis: All data were subjected to analysis of variance (ANOVA) with sums of squares partitioned to reflect trial effects using the SAS Software release $9.2^{17}$ and means were separated via Duncan multiple range test at $p=$ 0.05 .

\section{RESULTS AND DISCUSSION}

Carotenoid content in different cultivars of bitter melon: Three different carotenoids i.e., lutein, $\alpha$-carotene and $\beta$-carotene were determined among the 9 different cultivars collected from two locations (Japan and Philippines). There is a variation of carotenoids content among the bitter melon cultivars (Table-1). The carotenoid content differed significantly among the cultivars from two locations (Table-1). In general, the carotenoid content in the Philippines cultivar was higher than in the Japanese ones. Among the different cultivars, Galaxy from Philippines contained the highest amount of carotenoids among 9 cultivars and on the other hand, melons of the Japanese cultivar Kyushu contained the lowest amount of carotenoids. Significantly there is no variation of lutein content in the three cultivars i.e., Galaxy and Sta Monica from Philippines and Nikko from Japan and they contained the highest amount of lutein. The levels of lutein in the Galaxy cultivar were 2.9 and 2.8 times higher than Kyushu and Trident 357 cultivars, respectively. The variation of $\alpha$-carotene and $\beta$ carotene between the highest and lowest content cultivars were much higher compared to lutein. The cultivar Galaxy from Philippines contaien the highest amount of both $\alpha$-carotene and $\beta$-carotene. This cultivar (Galaxy) contained were 3.7, 3.1 and 3.0-fold higher $\alpha$-carotene compared to Kyushu, Sta.Rita Strain. L and Peacock, respectively. The trend of $\beta$ carotene content was almost similar with that of $\alpha$-carotene content in the cultivars. The levels of $\beta$-carotene in the Galaxy cultivars were 3.9, 2.9 and 2.8 times higher than that of Kyushu, Sta. Rita Strain. L and Peacock, respectively.

This study has shown the levels of an important compound (carotenoid) in different cultivars of bitter melon from 2 locations. Carotenoid levels varied widely among the cultivars, so that some of them produced substantially higher levels of carotenoids than did others. Specially, the Galaxy cultivar from Philippines performed the best for higher accumulation of all kinds of carotnoid. Carotenoid content varied due to cultivars and geographical loactions were reported in some vegetables, carotenoid content varied in the skins than the flesh of both types of kohlrabi ${ }^{18}$. Our findings are in agreement with Habicht et al. ${ }^{19}$ where they reported that the levels of saponin, linoleic and linolenic varied among the cultivars of bitter melon. They found that white bitter gourd varieties were found to contain significantly lower saponin concentrations $(0.25 \%)$ compared to green varieties $(0.67 \%)$. Differences regarding these findings may result from differences regarding the extraction methods and the different varieties investigated as Oishi et al..$^{20}$ do not provide any information about variety or plant part used. But, the results are of the same magnitude indicating that bitter gourd is a vegetable rich in saponins. For

TABLE-1

CAROTENOID CONTENTS IN DIFFERENT CULTIVARS OF Momordica charantia

\begin{tabular}{clccc}
\hline \multirow{2}{*}{ Country } & \multicolumn{1}{c}{ Cultivar } & \multicolumn{3}{c}{ Carotenoid $(\mu \mathrm{g} / \mathrm{g}$ dry weight) } \\
\cline { 3 - 5 } & Erabu & Lutein & $\alpha$-Carotene & $\beta$-Carotene \\
\hline \multirow{3}{*}{ Japan } & Kyushu & $8.09 \mathrm{c}$ & $0.50 \mathrm{~d}$ & $0.89 \mathrm{c}$ \\
& Nikko & $4.77 \mathrm{e}$ & $0.35 \mathrm{f}$ & $0.46 \mathrm{f}$ \\
& Peacock & $13.06 \mathrm{a}$ & $0.49 \mathrm{~d}$ & $0.73 \mathrm{~d}$ \\
& Galaxy & $7.75 \mathrm{c}$ & $0.43 \mathrm{e}$ & $0.64 \mathrm{e}$ \\
\hline \multirow{3}{*}{ Philippines } & $13.65 \mathrm{a}$ & $1.28 \mathrm{a}$ & $1.78 \mathrm{a}$ \\
& Sta Monica & $13.40 \mathrm{a}$ & $0.94 \mathrm{~b}$ & $1.41 \mathrm{~b}$ \\
& Sta. Rita Strain L. & $5.88 \mathrm{~d}$ & $0.42 \mathrm{e}$ & $0.66 \mathrm{e}$ \\
& Trident 357 & $4.80 \mathrm{e}$ & $0.46 \mathrm{de}$ & $0.63 \mathrm{e}$ \\
& Verde Buenas & $11.11 \mathrm{~b}$ & $0.63 \mathrm{c}$ & $1.35 \mathrm{~b}$ \\
\hline
\end{tabular}

Mean values (mean of three replicates with three samples from each replicate) indicated by the same letter in a column do not differ significantly at $5 \%$ level (Duncan multiple range test). 
example charantin is one important steroidal saponin in the bitter gourd with $\beta$-sitosterol and stigmasterol as aglycones ${ }^{21,22}$.

Because carotenoid has been used as a precursor of vitamin A, cultivars such as Galaxy could be used as a food supplement to supplement the carotenoid requirements for lower income people in developing countries. From the present research work, it is concluded that the bitter melon cultivar Galaxy from Philippines are good source of carotenoid.

\section{ACKNOWLEDGEMENTS}

This study was supported by research funds from Dong-A University, Korea, 2014.

\section{REFERENCES}

1. E. Basch, S. Gabardi and C. Ulbricht, Am. J. Health Syst. Pharm., 60, 356 (2003).

2. M.B. Krawinkel and G.B. Keding, Nutr. Rev., 64, 331 (2006)

3. W.T. Cefalu, J. Ye and Z.Q. Wang, Endocr. Metab. Immune Disord. Drug Targets, 8, 78 (2008).

4. L. Leung, R. Birtwhistle, J. Kotecha, S. Hannah and S. Cuthbertson, Br. J. Nutr., 102, 1703 (2009).

5. R. Nahas and M. Moher, Can. Fam. Physician, 55, 591 (2009).

6. R. Zafar and Neerja, Hamdard Med., 34, 49 (1991).
7. T.B. Ng, W.Y. Chan and H.W. Yeung, Gen. Pharmacol., 23, 579 (1992).

8. P. Scartezzini and E. Speroni, J. Ethnopharmacol., 71, 23 (2000).

9. J.K. Grover and S.P. Yadav, J. Ethnopharmacol., 93, 123 (2004).

10. N. Beloin, M. Gbeassor, K. Akpagana, J. Hudson, K. de Soussa, K. Koumaglo and J.T. Arnason, J. Ethnopharmacol., 96, 49 (2005).

11. S.A.R. Paiva and R.M. Russell, J. Am. Coll. Nutr., 18, 426 (1999)

12. J. Penuelas and S. Munne-Bosch, Trends Plant Sci., 10, 166 (2005).

13. C.A. Howitt and B.J. Pogson, Plant, Cell Environ., 29, 435 (2006).

14. IMFNB, $\beta$-carotene and Other Carotenoids: Dietary Reference Intakes for Vitamin C, Vitamin E, Selenium and Carotenoids, p. 325 (2000).

15. A. Mercandate and R. Amaya, J. Food Sci., 25, 213 (1990).

16. C. Yamini, N. Ranjana, Y. Chaturvedi and R. Nagar, J. Food Sci., 56, 127 (2001)

17. SAS, The Little SAS Book for Enterprise Guide 4.2. 294-295. Statistical Analysis Systems Institute, Cary, NC, USA (2010).

18. W.T. Park, J.K. Kim, S. Park, S.W. Lee, X. Li, Y.B. Kim, M.R. Uddin, N.I. Park, S.J. Kim and S.U. Park, J. Agric. Food Sci., 60, 8111 (2012).

19. S.D. Habicht, V. Kind, S. Rudloff, C. Borsch, A.S. Mueller, J. Pallauf, R. Yang and M.B. Krawinkel, Food Chem., 126, 172 (2011).

20. Y. Oishi, T. Sakamoto, H. Udagawa, H. Taniguchi, K. KobayashiHattori, Y. Ozawa and T. Takita, Biosci. Biotechnol. Biochem., 71, 735 (2007).

21. A.M. Dans, M.V. Villarruz, C.A. Jimeno, M.A. Javelosa, J. Chua, R. Bautista and G.G.B. Velez, J. Clin. Epidemiol., 60, 554 (2007).

22. L. Harinantenaina, M. Tanaka, S. Takaoka, M. Oda, O. Mogami, M. Uchida and Y. Asakawa, Chem. Pharm. Bull. (Tokyo), 54,1017 (2006). 